\title{
Generalizations of an asymptotic stability theorem of Bahyrycz, Páles and Piszczek on Cauchy differences to generalized cocycles
}

\author{
Árpád Száz
}

\begin{abstract}
We prove some straightforward analogues and generalizations of a recent asymptotic stability theorem of A. Bahyrycz, Zs. Páles and M. Piszczek on Cauchy differences to semi-cocycles and pseudo-cocycles introduced in a former paper by the present author.
\end{abstract}

Mathematics Subject Classification (2010): 39B52, 39B82, 20K99, 22 A99.

Keywords: Cauchy-differences, generalized cocycles, asymptotic and hyper stabilities.

\section{Introduction}

The first results on a certain stability property of the Cauchy functional equation

$$
f(x+y)=f(x)+f(y)
$$

were proved by Pólya and Szegö [63, p. 171 ] in 1925 and Hyers [38] in 1941.

In particular, Pólya and Szegö proved the following statement in two rather difficult ways.

Theorem 1.1. Suppose that the number sequence $a_{1}, a_{2}, a_{3}, \ldots$ satisfies the condition

$$
a_{m}+a_{n}-1<a_{m+n}<a_{m}+a_{n}+1 .
$$

Then, there exists the limit

$$
\lim _{n \rightarrow \infty} \frac{a_{n}}{n}=\omega
$$

and even more $\omega$ is finite, and for all $n=1,2,3, \ldots$ there holds

$$
\omega n-1<a_{n}<\omega n+1 \text {. }
$$


Remark 1.2. The significance of this theorem was not recognized either by Pólya and Szegö or the mathematical community for a long time. It was first cited by Kuczma $[50$, p. 424] in 1985 at the suggestion of R. Ger. However, in contrast to $[5,11]$, several authors have still not been mentioning it.

By R. Ger [34, p. 4] and some communications with Ger and M. Laczkovich, his attention to this theorem was first drawn by Laczkovich, at an undetectable conference, who indicated that the real-valued particular case of Hyers's stability theorem can be derived from it. His proof, reconstructed with the help of Ger and M. Sablik, can be found in $[77$, p. 633].

Hyers, giving a partial answer to a general problem proposed by S. M. Ulam before the Mathematics Club of the University of Wisconsin in 1940, proved the following fundamental theorem in a quite simple way.

An obvious generalization of his theorem to a function of a commutative semigroup to a Banach space [30, p. 216] already includes Theorem 1.1. Moreover, by Remark 1.2 and [30, Theorem 3], the two theorems are actually equivalent.

Theorem 1.3. Let $E$ and $E^{\prime}$ be Banach spaces and let $f(x)$ be a $\delta$-linear transformation of $E$ into $E^{\prime}$. Then the limit $l(x)=\lim _{n \rightarrow \infty} f\left(2^{n} x\right) / 2^{n}$ exists for each $x$ in $E, l(x)$ is a linear transformation, and the inequality $\|f(x)-l(x)\| \leq \delta$ is true for all $x$ in $E$.

Remark 1.4. Moreover, Hyers also stated that $l(x)$ is the only linear transformation satisfying this inequality.

Here, in contrast to the recent terminology, Hyers used the term "linear" instead of "additive". Thus, his $\delta$-linearity of $f(x)$ means only that

$$
\|f(x+y)-f(x)-f(y)\| \leq \delta \text { for all } x, y \in E .
$$

Now, because of $l(n x)=n l(x)$, we also have

$$
\|f(n x) / n-l(x)\|=\|f(n x)-l(n x)\| / n \leq \delta / n
$$

for all $n \in \mathbb{N}$ and $x \in E$. Therefore, analogously to Theorem 1.1 of Pólya and Szegö, we can also state that $l(x)=\lim _{n \rightarrow \infty} f(n x) / n$ for all $x \in X$.

The above basic Theorem 1.3 of Hyers has been generalized, in one direction, by Aoki [6], Th. M. Rassias [65], J. M. Rassias [64], Gǎvruţă [32] (see also [43]), and in other directions by several further mathematicians.

Moreover, some counterexamples, showing the necessity of certain extra assumptions in the corresponding stability theorems, such as commutativity and completeness for instance, have also been provided.

The interested reader can get a rapid overview on this enormous subject by consulting some of the numerous survey papers $[39,34,31,66,67,68,69,82,56,70$, $71,23,21,12,17,57]$ and the fundamental books $[40,44,22,49,46]$.

The above works show that some local, restricted, asymptotic, super and hyper stability results have also been proved for the Cauchy equation. Moreover, some close relationships with invariant means, sandwich and fixed point theorems have also been established. And, quite early, some set-valued generalizations have also been given. 
These set-valued generalizations show that Hyers's stability theorem is actually an additive selection theorem for a subadditive relation. Therefore, some of its generalizations should be derived from those of the Hahn-Banach extension theorems. (For some ideas in this respect, see [22, Chapter 34] and [35, 77, 36].)

However, it is now more important to note that recently Bahyrycz, Páles and Piszczek [9] have proved a new type, asymptotic stability theorem for the Cauchy functional equation by using metric Abelian groups instead of normed spaces.

They called a triple $(X,+, d)$ to be a metric abelian group if $(X,+)$ is an abelian group and $d$ is a translation invariant metric on $X$ in the sense that

$$
d(x+z, y+z)=d(x, y) \text { for all } x, y, z \in X .
$$

In this case, they defined $\|x\|_{d}=d(x, 0)$ for all $x \in X$, and noticed that \|\|$_{d}$ is an even subadditive function function on $X$ which is not, in general, even 2-homogeneous.

In $[74,83,13,48]$, metric groups and groupoids have been used in different senses. From Remarks 3.1 of $[83,13]$, we can see that a metric $d$ on a group $X$ is translation invariant if and only if $d(x+y, z+w) \leq d(x, z)+d(y, w)$ for all $x, y, z, w \in X$.

Thus, if in particular $d$ is a translation invariant metric on a group $X$, then the addition in $X$ is continuous. Moreover, in this case we can also note that

$$
d(-x,-y)=d(0, x-y)=d(y, x)=d(x, y) \text { for all } x, y \in X .
$$

Thus, in particular the inversion in $X$ is also continuous.

In particular, in [9], Bahyrycz, Páles and Piszczek have proved the following asymptotic stability theorem.

Theorem 1.5. Let $(X,+, d)$ and $(Y,+, \rho)$ be metric abelian groups such that $X$ is unbounded by $d$. Let $\varepsilon \geq 0$ and assume that $f: X \rightarrow Y$ possesses the asymptotic stability property

$$
\limsup _{\min \left(\|x\|_{d},\|y\|_{d}\right) \rightarrow \infty}\|f(x+y)-f(x)-f(y)\|_{\rho} \leq \varepsilon,
$$

then

$$
\|f(x+y)-f(x)-f(y)\|_{\rho} \leq 5 \varepsilon \quad \text { for all } \quad x, y \in X .
$$

Remark 1.6. Moreover, by taking $\varepsilon>0$ and $x_{0} \in X \backslash\{0\}$, and defining

$$
f\left(x_{0}\right)=3 \varepsilon \quad \text { and } \quad f(x)=\varepsilon \quad \text { for } \quad x \in X \backslash\left\{x_{0}\right\},
$$

they have also proved that 5 is the smallest possible constant in the above theorem.

The most closely related related results to Theorem 1.5 are [52, Theorem 1] of Losonczi with the same constant 5, and the results of Jung, Moslehian, and Sahoo $[45,47]$ and Chung $[18,19,20]$ with some other natural constants in the concluded estimates.

The origins of these investigations go back to Skof [72, 73], Hyers, Isac and Rassias [41] and Găvruţă [33]. (See also [54].) In the real-valued case, Volkmann [84] proved the best estimate.

From Theorem 1.5, by taking $\varepsilon=0$, Bahyrycz, Páles and Piszczek could immediately derive the following asymptotic hyperstability result. 
Corollary 1.7. Let $(X,+, d)$ and $(Y,+, \rho)$ be metric abelian groups such that $X$ is unbounded by $d$. If $f: X \rightarrow Y$ satisfies

$$
\limsup _{\min \left(\|x\|_{d},\|y\|_{d}\right) \rightarrow \infty}\|f(x+y)-f(x)-f(y)\|_{\rho}=0,
$$

then

$$
f(x+y)=f(x)+f(y), \quad x, y \in X .
$$

Hyperstability results, for the Cauchy equation and its generalizations, have also been proved by Maksa and Páles [53], Najati and Rassias [59], Alimohammady and Sadeghi [3], and Brzdek [14, 15, 16], Piszczek [61, 62], Almahalebi, Charifi and Kabbaj [4], Bahyrycz and Olko [7, 8], Aiemsomboon and Sintunavarat [1, 2], Molaei, Najati and Park [55, 58].

Moreover, several interesting asymptotic stability and hyperstability theorems for additive functions have also been proved by using some other functional equations than the Cauchy and generalized Cauchy ones.

In the present paper, we shall improve and generalize Theorem 1.5 of Bahyrycz, Páles and Piszczek. For this, we shall use preseminormed groups instead of the metric ones. Moreover, we shall use generalized cocycles introduced in [78], instead of the Cauchy difference

$$
F(x, y)=f(x+y)-f(x)-f(y)
$$

Some basic definitions and results on these fundamental objects, which are certainly unfamiliar to the reader, will be briefly laid out in the next preparatory section.

\section{A few basic facts on preseminorms and generalized cocycles}

Motivated by the corresponding definitions of $[76,80]$ and the proofs of our forthcoming theorems, an even subadditive function \|\| of a group $X$ to $\mathbb{R}$ will be called a preseminorm on $X$.

Thus, under the notation $\|x\|=\|\|(x)$, we have $\|0\|=\|0+0\| \leq\|0\|+\|0\|$, and thus $0 \leq\|0\|$. And more generally, $\|0\|=\|x+(-x)\| \leq\|x\|+\|-x\|=2\|x\|$, and thus $0 \leq\|x\|$ for all $x \in X$.

Therefore, if $\|0\| \neq 0$, then by defining $\|x\|^{\star}=0$ for $x=0$, and $\|x\|^{\star}=\|x\|$ for $x \in X \backslash\{0\}$, we can obtain a new preseminorm \|\|$^{\star}$ on $X$ such that $\|0\|^{\star}=0$ already holds.

By using induction and the corresponding definitions, we can also easily see that $\|n x\| \leq n\|x\|$, and thus $\|(-n) x\|=\|n(-x)\| \leq n\|-x\|=n\|x\|$ for all $n \in \mathbb{N}$ and $x \in X$.

Therefore, the preseminorm \|\| may be naturally called a seminorm if $n\|x\| \leq$ $\|n x\|$ for all $x \in X$. Namely, thus we have $\|k x\|=|k|\|x\|$ for all $x \in X$ and $k \in \mathbb{Z} \backslash\{0\}$. (If $\|0\|=0$, then this also holds for $k=0$.)

Note that a nonzero seminorm cannot be bounded. While, if \|\| is a seminorm (preseminorm) on $X$, then for instance the function defined by $\|x\|^{*}=\min \{1,\|x\|\}$ for all $x \in X$ is a bounded preseminorm on $X$. 
Now, a seminorm (preseminorm) \|\| on $X$ may be naturally called a norm (prenorm) if $\|x\|=0$ implies $x=0$ for all $x \in X$. If $X=\mathbb{Z} x$ for all $x \in X \backslash\{0\}$, then each nonzero preseminorm on $X$ is a prenorm.

In [79, Remark 3.14], with the help of G. Horváth, it was proved that the latter condition is equivalent to the requirement that the cardinality of $X$ is prime, or equivalently $X$ has no nontrival proper subgroup.

Now, for instance, an ordered pair $X(\|\|)=(X,\|\|)$ consisting a group $X$ and a preseminorm \|\| on $X$, may be naturally called a preseminormed group. And, we may simply write $X$ instead of $X(\|\|)$.

If $X$ is a preseminormed group, then because of the subadditivity and evenness of the corresponding preseminorm, for any $x, y \in X$, we have

$$
\|x\|=\|x+y-y\| \leq\|x+y\|+\|-y\|=\|x+y\|+\|y\|
$$

and

$$
\|y\|=\|-x+x+y\| \leq\|-x\|+\|x+y\|=\|x\|+\|x+y\| .
$$

Therefore,

$$
|\|x\|-\|y\|| \leq\|x+y\| \quad \text { and } \quad|\|x\|-\|y\||=|\|x\|-\|-y\|| \leq\|x-y\| .
$$

However, it is now more important to note that Bahyrycz, Páles and Piszczek, in the proof of their Theorem 1.5, have used, but not explicitly stated, the equality

$$
\begin{aligned}
& f(x+y)-f(x)-f(y)=f(x-u)+f(u)-f(x) \\
& \quad+f(y-v)+f(v)-f(y)+f(x+y-u-v)-f(x-u)-f(y-v) \\
& \quad+f(u+v)-f(u)-f(v)+f(x+y)-f(x+y-u-v)-f(u+v) .
\end{aligned}
$$

In a former paper [78], by using the Cauchy difference (1.2), we have noticed that, instead of equation (2.1), it is more convenient to consider the equation

$$
\begin{aligned}
F(x, y) & =F(u, v)-F(x-u, u)-F(y-v, v) \\
& +F(x-u, y-v)+F(x+y-u-v, u+v) .
\end{aligned}
$$

Namely, thus Theorem 1.5 can be easily extended to the solutions of (2.2). Moreover, we can prove that every symmetric cocycle $F$ on $X$ to $Y$ is a solution of this equation.

That is, if $F$ is a function of $X^{2}$ to $Y$ such that $F(x, y)=F(y, x)$ and

$$
F(x, y)+F(x+y, z)=F(x, y+z)+F(y, z)
$$

for all $x, y, z \in X$, then (2.2) also holds for all $x, y, u, v \in X$.

It is well-known that every Cauchy-difference is a symmetric cocycle. Moreover, Davison and Ebanks [24, Lemma 2] have proved that if $F$ is a symmetric cocycle on $X$ to $Y$, then

$$
F(x+y, u+v)=F(x+u, y+v)+F(x, u)+F(y, v)-F(x, y)-F(u, v)
$$

also holds for all $x, y, u, v \in X$.

At first seeing, I considered equations (2.2) and (2.4) to be very similar, but still quite independent. However, Gyula Maksa, my close colleague, has noticed that they are actually equivalent. 
Namely, (2.4) can be immediately derived from (2.2) by replacing $x$ by $x+u$ and $y$ by $y+v$. And conversely, (2.2) can be immediately derived from (2.4) by replacing $x$ by $x-u$ and $y$ by $y-v$. Thus, equation (2.1) is a consequence of (2.4) too.

Inspired by the above observations, in our former paper [78], we have also considered the more difficult equations

$$
\begin{aligned}
F(x, y) & +F(u, y+v)+F(x+y, u+v) \\
& =F(x, u)+F(y, u+v)+F(x+u, y+v)
\end{aligned}
$$

and

$$
\begin{aligned}
F(x, y) & +F(x-u, u)+F(y-v, u)+F(y-v, v) \\
& =F(u, v)+F(u, y-v)+F(x-u, y-v)+F(x+y-u-v, u+v) .
\end{aligned}
$$

Note that if in particular $F$ is symmetric, then equation (2.6) is equivalent to (2.2), which is in turn equivalent to (2.4). Moreover, it can be easily shown that if $F$ is additive in its second variable, then equations (2.5) and (2.6) are also equivalent.

In our former paper [78], by using some more difficult computations, we have also proved that equations (2.5) and (2.6) are also natural generalizations of (2.3) too. Therefore, their solutions may be naturally called semi-cocycles and pseudo-cocycles, respectively.

In the light of the above observations, it seems to be a reasonable research program to extend some of the basic theorems on cocycles to these generalized cocycles. And, to establish some deeper relationships among the various generalizations of cocycles mentioned in [78].

However, in the sequel, we shall only prove some straightforward analogues and generalizations of Theorem 1.5 to semi-cocycles and pseudo-cocycles.

\section{Analogues of Theorem 1.5 for generalized cocycles}

Notation 3.1. In the sequel, we shall assume that $F$ is a function of an unbounded, commutative preseminormed group $X$ to a commutative preseminormed group $Y$.

Remark 3.2. Note that now, by defining

$$
(x, y)+(u, v)=(x+u, y+v)
$$

and

$$
\|(x, y)\|=\|x\| \vee\|y\|=\max \{\|x\|,\|y\|\}
$$

for all $x, y, u, v \in X$, the set $X^{2}$ can also be turned into an unbounded commutative preseminormed group.

Thus, by using a more simple argument than that used by Bahyrycz, Páles and Piszczek in [9], we can prove the following natural analogue of Theorem 1.5.

Theorem 3.3. If $F$ is a semi-cocycle and

$$
\varepsilon=\varlimsup_{\|z\| \rightarrow+\infty}\|F(z)\|,
$$


then

for all $z \in X^{2}$.

$$
\|F(z)\| \leq 5 \varepsilon
$$

Proof. By the corresponding definitions, for any $\eta>\varepsilon$, we have

$$
\inf _{r>0} \sup _{\|z\|>r}\|F(z)\|<\eta \text {. }
$$

Therefore, there exists $r>0$ such that $\sup _{\|z\|>r}\|F(z)\|<\eta$, and thus

$$
\|F(z)\|<\eta
$$

for all $z \in X^{2}$ with $\|z\|>r$.

Hence, since $\|z\|=\left\|\left(z_{1}, z_{2}\right)\right\| \geq\left\|z_{i}\right\|$ for $i=1,2$, it is clear that in particular we have

$$
\|F(s, t)\|<\eta
$$

for all $s, t \in X$ with either $\|s\|>r$ or $\|t\|>r$.

Now, by taking $x, y \in X$ and using equation (2.5), we can see that

$$
\begin{aligned}
\|F(x, y)\| & =\| F(x, u)+F(y, u+v)-F(u, y+v) \\
& +F(x+u, y+v)-F(x+y, u+v) \| \\
& \leq\|F(x, u)\|+\|F(y, u+v)\|+\|F(u, y+v)\| \\
& +\|F(x+u, y+v)\|+\|F(x+y, u+v)\|<5 \eta
\end{aligned}
$$

whenever for instance $u, v \in X$ such that

$$
\|u\|>r, \quad\|u+v\|>r, \quad\|x+u\|>r .
$$

Therefore, if such $u$ and $v$ exist, then

$$
\|F(x, y)\|<5 \eta, \quad \text { and thus } \quad\|F(x, y)\| \leq 5 \varepsilon
$$

Now, to complete the proof, it remains to show only that the required $u$ and $v$ exist. For this, we can note that, because of the assumed unboundedness of $X$, there exist $u, v \in X$ such that

$$
\|u\|>r+\|x\| \quad \text { and } \quad\|v\|>r+\|u\| .
$$

Thus, we evidently have $\|u\|>r$. Moreover, by using the inequality $\|s+t\| \geq\|t\|-\|s\|$, we can also see that

$$
\|x+u\| \geq\|u\|-\|x\|>r+\|x\|-\|x\|=r
$$

and

$$
\|u+v\| \geq\|v\|-\|u\|>r+\|u\|-\|u\|=r .
$$

From equation (2.6) and the proof of Theorem 3.3, it is clear that we also have

Theorem 3.4. If $F$ is a pseudo-cocycle and

$$
\varepsilon=\varlimsup_{\|z\| \rightarrow+\infty}\|F(z)\|
$$

then

for all $z \in X^{2}$.

$$
\|F(z)\| \leq 7 \varepsilon
$$


Now, as an immediate consequence of the above two theorems, we can also state Corollary 3.5. If $Y$ is prenormed, $F$ is either a semi or pseudo cocycle, and

$$
\varlimsup_{\|z\| \rightarrow+\infty}\|F(z)\|=0
$$

then $F(z)=0$ for all $z \in X^{2}$.

Remark 3.6. However, from Theorems 3.3 and 3.4 we cannot get proper generalizations of Theorem 1.5. Therefore, in the next section we shall prove some modification and improvement of Theorem 3.4.

\section{Proper and partial generalizations of Theorem 1.5 to pseudo-cocycles}

Remark 4.1. Because of the condition of Theorem 1.5, in the sequel we shall also use the quantity

$$
\nVdash(x, y) \nVdash=\|x\| \wedge\|y\|=\min \{\|x\|,\|y\|\},
$$

for all $(x, y) \in X^{2}$ instead of the natural preseminorm considered in Remark 3.2. Thus, the function $\nVdash \nVdash$ is not a preseminorm on $X^{2}$. However, despite this, it can be well used to measure the magnitude of the points of $X^{2}$.

Moreover, it can as well be used to prove the following proper and partial generalizations of Theorem 1.5 to pseudo-cocycles. The proof of the first one is quite similar to the second one. Therefore, it will be omitted.

Theorem 4.2. If $F$ is a symmetric pseudo-cocycle and

$$
\varepsilon=\varlimsup_{\nVdash z \nVdash \rightarrow+\infty}\|F(z)\|,
$$

then

$$
\|F(z)\| \leq 5 \varepsilon
$$

for all $z \in X^{2}$.

The proof of the following theorem is again quite similar, but a little more readable, than the one given by Bahyrycz, Páles and Piszczek in [9].

Theorem 4.3. If $F$ is a pseudo-cocycle and

$$
\varepsilon=\varlimsup_{\nVdash z \nVdash \rightarrow+\infty}\|F(z)\|,
$$

then

$$
\|F(z)\| \leq 7 \varepsilon
$$

for all $z \in X^{2}$. 
Proof. By the corresponding definitions, for any $\eta>\varepsilon$, we have

$$
\inf _{r>0} \sup _{\nVdash z \nVdash>r}\|F(z)\|<\eta \text {. }
$$

Therefore, there exists $r>0$ such that $\sup _{\Varangle z ł>r}\|F(z)\|<\eta$, and thus

$$
\|F(z)\|<\eta
$$

for all $z \in X^{2}$ with $\nVdash z \nVdash>r$.

Hence, since $\nVdash z \nVdash=\nVdash\left(z_{1}, z_{2}\right) \nVdash=\min \left\{\left\|z_{1}\right\|,\left\|z_{2}\right\|\right\}$, it is clear that in particular we have

$$
\|F(s, t)\|<\eta
$$

for all $s, t \in X$ with $\|s\|>r$ and $\|t\|>r$.

Now, by taking $x, y \in X$ and using equation (2.6), we can see that

$$
\begin{aligned}
\|F(x, y)\| & =\| F(u, v)+F(u, y-v)-F(y-v, u) \\
& -F(x-u, u)-F(y-v, v)+F(x-u, y-v)+F(x+y-u-v, u+v) \| \\
& \leq\|F(u, v)\|+\|F(u, y-v)\|+\|F(y-v, u)\| \\
& +\|F(x-u, u)\|+\|F(y-v, v) \mid+\| F(x-u, y-v) \| \\
& +\|F(x+y-u-v, u+v)\|<7 \eta
\end{aligned}
$$

whenever $u, v \in X$ such that

$$
\begin{aligned}
& \|u\|>r, \quad\|v\|>r, \quad\|x-u\|>r, \quad\|y-v\|>r, \\
& \|u+v\|>r, \quad\|x+y-u-v\|>r .
\end{aligned}
$$

Therefore, if such $u$ and $v$ exist, then

$$
\|F(x, y)\|<7 \eta, \quad \text { and thus } \quad\|F(x, y)\| \leq 7 \varepsilon .
$$

Now, to complete the proof, it remains only to show that the required $u$ and $v$ exist. For this, following the arguments given [9], we can note that because of the assumed unboundedness of $X$ there exist $u, v \in X$ such that

$$
\|u\|>r+\|x\| \quad \text { and } \quad\|v\|>r+\|x\|+\|y\|+\|u\| .
$$

Thus, we evidently have $\|u\|>r$ and $\|v\|>r$. Moreover, by using the facts that $\|s+t\| \geq\|t\|-\|s\|$ and $\|-t\|=\|t\|$, we can also see that

$$
\begin{gathered}
\|x-u\| \geq\|u\|-\|x\|>r+\|x\|-\|x\|=r, \\
\|y-v\| \geq\|v\|-\|y\|>r+\|x\|+\|y\|+\|u\|-\|x\|=r+\|y\|+\|u\| \geq r,
\end{gathered}
$$

and

$$
\begin{gathered}
\|u+v\| \geq\|v\|-\|u\|>r+\|x\|+\|y\|+\|u\|-\|u\|=\underline{r+\|x\|+\|y\|} \geq r, \\
\|x+y-u-v\| \geq\|u+v\|-\|x+y\|>r+\|x\|+\|y\|-\|x\|-\|y\|=r .
\end{gathered}
$$

Namely, because of the inequality $\|x\|+\|y\| \geq\|x+y\|$, we also have

$$
-\|x+y\| \geq-\|x\|-\|y\| .
$$

From this theorem, we can immediately derive 
Corollary 4.4. If $Y$ is prenormed, $F$ is a pseudo-cocycle and

$$
\varlimsup_{\nVdash z \nVdash \rightarrow+\infty}\|F(z)\|=0,
$$

then $F(z)=0$ for all $z \in X^{2}$.

Remark 4.5. Recall that a Cauchy-difference is a symmetric cocycle. Moreover, a cocycle is both a semi-cocycle and a pseudo-cocycle.

Therefore, in Theorem 4.2, F may, in particular, be a Cauchy-difference or a symmetric cocycle. While, in Theorems $3.3,3.4$ and 4.3 and their corollaries, $F$ may already be an arbitrary cocycle.

\section{Some supplementary notes}

Remark 5.1. If for instance $f$ is a function of $X$ to $Y$ and $\varepsilon \geq 0$ such that there exists $r>0$ such that

$$
\|f(x+y)-f(x)-f(y)\| \leq \varepsilon
$$

for all $x, y \in X$ with $\|x\|,\|y\|>r$, then by Remark 4.1 and the definition of the upper limit we have

$$
\varlimsup_{\nVdash(x, y) \nVdash \rightarrow+\infty}\|f(x+y)-f(x)-f(y)\| \leq \varepsilon .
$$

Thus, by Remark 4.5 and Theorem 4.2, we can state that

$$
\|f(x+y)-f(x)-f(y)\| \leq 5 \varepsilon
$$

for all $x, y \in X$. Therefore, Theorem 1.5 follows from Theorem 4.2.

Now, if in particular $Y$ is the additive group of a Banach space, then by using a slight generation of Theorem 1.3 we can also state that there exists an additive function $g$ of $X$ to $Y$ such that

$$
\|f(x)-g(x)\| \leq 5 \varepsilon
$$

for all $x \in X$.

Remark 5.2. While, if $f$ is an arbitrary and $g$ is an additive function of $X$ to $Y$ such that

$$
\|f(x)-g(x)\| \leq 5 \varepsilon
$$

for all $x \in X$, then we can only state that

$$
\begin{aligned}
\|f(x+y)-f(x)-f(y)\| & =\|f(x+y)-g(x+y)+g(x)-f(x)+g(y)-f(y)\| \\
& \leq\|f(x+y)-g(x+y)\|+\|g(x)-f(x)\|+\|g(y)-f(y)\| \\
& \leq 15 \varepsilon
\end{aligned}
$$

for all $x, y \in X$. Therefore, Theorem 1.5 is sharper than the one derivable from the usual asymptotic stability theorems.

This clearly reveal that the corresponding theorems on restricted and assymptotic stabilities have to split into two parts. This idea is also apparent from the proofs of those theorems. 
Remark 5.3. Concerning our former results, it is also worth mentioning that in Theorems 3.3 and 3.4 and Corollary 3.5, instead of the "supremum preseminorm"

$$
\|(x, y)\|=\|(x, y)\|_{\infty}=\max \{\|x\|,\|y\|\}
$$

considered in Remark 3.2, we may also naturally use an " $L_{p}$-preseminorm", defined by

$$
\|(x, y)\|=\|(x, y)\|_{p}=\left(\|x\|^{p}+\|y\|^{p}\right)^{1 / p}
$$

for some $1 \leq p<+\infty$ and all $(x, y) \in X^{2}$. Namely, this also has the important property that $\left\|z_{i}\right\| \leq\|z\|$ for $i=1,2$, whenever $z \in X^{2}$.

Remark 5.4. Moreover, we can also note that $\|z\| \geq \nVdash z \nVdash$ for all $z \in X^{2}$. Therefore,

$$
\{F(z): \nVdash z \nVdash>r\} \subseteq\{F(z):\|z\|>r\},
$$

and thus

for all $r>0$. Consequently,

$$
\sup _{\sharp z \nVdash>r}\|F(z)\| \leq \sup _{\|z\|>r}\|F(z)\|
$$

$$
\varlimsup_{\nVdash z \nVdash \rightarrow+\infty}\|F(z)\|=\inf _{r>0} \sup _{\nVdash z \nVdash>r}\|F(z)\| \leq \inf _{r>0} \sup _{\|z\|>r}\|F(z)\|=\varlimsup_{\|z\| \rightarrow+\infty}\|F(z)\| .
$$

Therefore, the results obtained with $\|$ || are usually much weaker than that obtained with $\nVdash \nVdash$. However, the former ones are, in a certain sense, still more natural since $\nVdash \nVdash$ is not a preseminorm on $X^{2}$.

\section{Suggestions for further investigations}

Cauchy differences, in the theory of functional equations, were first characterized by Kurepa [51] and Erdős [29] with the help of the equation

$$
F(x, y)+F(x+y, z)=F(x, y+z)+F(y, z)
$$

(For the algebraic origins and several further developments on this cocycle equation, see the book [75] by Stetkaer.)

Quadratic differences were first characterized by Székelyhidi [81] with the help of the equation

$$
F(x+y, z)+F(x-y, z)-2 F(y, z)=F(x, y+z)+F(x, y-z)-2 F(x, y) .
$$

(For some closely related results, see also Ebanks and $\mathrm{Ng}[25,28]$.)

Some results on equations (6.1) and (6.2) were extended by Páles [60] to the more attractive equation

$$
F(x, y)+\frac{1}{n} \sum_{i=1}^{n} F\left(x+\phi_{i}(y), z\right)=\frac{1}{n} \sum_{i=1}^{n} F\left(x, y+\phi_{i}(z)\right)+F(y, z) .
$$

(Later, this important equation has only been studied by Maksa and Páles [53].)

Recently, Leibniz differences has been characterized by Ebanks [27] with the help of the equation

$$
F(x y, z)+x F(x, y)=F(x, y z)+x F(y, z)
$$


(For some earlier results, see Jessen, Karpf and Thorup [42], Ebanks [26] and Gselmann and Páles [37].)

Moreover, affine differences has been characterized by Boros [10] with the help of the equation

$$
\begin{aligned}
F(s, r x+(1-r) y, t x+(1-t) y) & =F(s r+(1-s) t, x, y) \\
& -s F(r, x, y)-(1-s) F(t, x, y) .
\end{aligned}
$$

Thus, it is certainly true that several further important differences, such as for instance the Jensen one, can also be characterized with the help of some functional equations containing a little more variables than the corresponding differences.

Therefore, it seems to be a reasonable research program to prove some counterparts of the results of Bahyrycz, Páles and Piszczek [9] and the present author for such equations and their generalizations. First of all, some analogues and generalizations of [9, Theorem 2] could be proved.

Added in Proof. The original version of this paper (Tech. Rep., Inst. Math., Univ. Debrecen 20016/2, 12 pp.) was planned to be published in the Proceedings of the Conference on Ulam's Type Stability, 2016, Cluj-Napoca, Romania. However, it was later submitted to the present journal.

Here, to improve the presentation, some useful changes have been suggested by the referee and the editor. In particular, according to a general regulation of the journal, all items of References have to be cited in the text. Therefore, my original manuscript has been substantially rewritten.

\section{References}

[1] Aiemsomboon, L., Sintunavarat, W., On generalized hyperstability of a general linear equation, Acta Math. Hungar., 149(2016), 413-422.

[2] Aiemsomboon, L., Sintunavarat, W., A note on the generalized hyperstability of the general linear equation, Bull. Aust. Math. Soc., 96(2017), 263-273.

[3] Alimohammady, M., Sadeghi, A., On the aymptotic behavior of Pexiderized additive mapping on semigroups, Fasc. Math., 49(2012), 5-14.

[4] Almahalebi, M., Charifi, A., Kabbaj, S., Hyperstability of a Cauchy functional equation, J. Nonlinear Anal. Optim., 6(2015), 127-137.

[5] Anderson, R.M., "Almost" implies "near", Trans. Amer. Math. Soc., 296(1986), 229237.

[6] Aoki, T., On the stability of the linear transformation in Banach spaces, J. Math. Soc. Japan, 2(1950), 491-495.

[7] Bahyrycz, A., Hyperstability of some functional equation on restricted domain: Direct and fixed point method, Bull. Iranian Math. Soc., 42(2016), 959-974.

[8] Bahyrycz, A., Olko, J., Hyperstability of general linear functional equation, Aequationes Math., 90(2016), 527-540.

[9] Bahyrycz, A., Páles Zs., Piszczek, M., Asymptotic stability of the Cauchy and Jensen functional equations, Acta Math. Hungar., 150(2016), 131-141. 
[10] Boros, Z., A characterization of affine differences on intervals, Report of Meeting, The Seventeenth Katowice-Debrecen Winter Seminar, Zakopane (Poland), February 1-4, 2017, Ann. Math. Sil., 31(2017), 188-189.

[11] Boualem, H., Brouzet, R., On what is the almost-near principle, Amer. Math. Monthly, 119(2012), 381-397.

[12] Brillouët-Bellout, N., Brzdek, J., Ciepliński, K., On some recent developments in Ulam's type stability, Abstr. Appl. Anal., 2012 (2012), 41 pp.

[13] Brzdek, J., On a method of proving the Hyers-Ulam stability of functional equations on restricted domains, Aust. J. Math. Anal. Appl., 6(2009), 10 pp.

[14] Brzdek, J., Hyperstability of the Cauchy equation on restricted domains, Acta Math. Hungar., 141(2013), 58-67.

[15] Brzdek, J., Remarks on hyperstability of the Cauchy functional equation, Aequationes Math., 86(2013), 255-267.

[16] Brzdek, J., A hyperstability result for Cauchy equation, Bull. Aust. Math. Soc., 89(2014), 33-40.

[17] Brzdek, J., Ciepliński, K., Hyperstability and superstability, Abstr. Appl. Anal., Vol. 2013, 13 pp.

[18] Chung, J.-Y., Stability of functional equations on restricted domains in a group and their asymptotic behaviors, Comput. Appl. Math., 60(2010), 2653-2665.

[19] Chung, J.-Y., Stability of a conditional Cauchy equation, Aequationes Math., 83(2012), 313-320.

[20] Chung, J.-Y., Stability of a conditional Cauchy equation on a set of measure zero, Aequationes Math., 87(2014), 391-400.

[21] Ciepliński, K., Applications of fixed point theorems to the Hyers-Ulam stability of functional equations - A survey Ann. Funct. Anal., 3(2012), 151-164.

[22] Czerwik, S., Functional Equations and Inequalities in Several Variables, World Scientific, London, 2002.

[23] Czerwik, S., Król, K., Ulam stability of functional equations, Aust. J. Math. Anal. Appl., 6(2009), 15 pp.

[24] Davison, T.M.K., Ebanks, B.R., Cocycles on cancellative semigroups, Publ. Math. Debrecen, 46(1995), 137-147.

[25] Ebanks, B.R., Differentiable solutions of a functional equation of Székelyhidi, Util. Math., 36(1989), 197-199.

[26] Ebanks, B.R., On some functional equations of Jessen, Karpf, and and Thorup, Math. Scand., 44(1979), 231-234.

[27] Ebanks, B.R., On Leibniz differences, Publ. Math. Debrecen, 91 (2017), 143-151.

[28] Ebanks, B.R., Ng, C.T., Characterizations of quadratic differences, Publ. Math. Debrecen, 48(1996), 89-102.

[29] Erdős, J., A remark on the paper "On some functional equations" by S. Kurepa, Glas. Mat. Ser. III, 14(1959), 3-5.

[30] Forti, G.L., The stability of homomorphisms and amenability, with applications to functional equations, Abh. Math. Semin. Univ. Hambg., 57(1987), 215-226.

[31] Forti, G.L., Hyers-Ulam stability of functional equations in several variables, Aequationes Math., 50(1995), 143-190. 
[32] Găvruţă, P., A generalization of the Hyers-Ulam-Rassias stability of approximately additive mappings, J. Math. Anal. Appl., 184(1994), 431-436

[33] Găvruţă, P., On the Hyers-Ulam-Rassias asymptotic stability of mappings, Bul. Ştiinţ. Univ. Politeh. Timiş. Ser. Mat. Fiz., 41(1996), 48-51.

[34] Ger, R., A survey of recent results on stability of functional equations, Proceedings of the 4th International Conference on Functional Equations and Inequalities, Pedagogical University of Cracow, 1994, 5-36.

[35] Glavosits, T., Száz, Á., A Hahn-Banach type generalization of the Hyers-Ulam theorem, An. Ştiinţ. Univ. "Ovidius" Constanţa Ser. Mat., 19(2011), 139-144.

[36] Glavosits, T., Száz, Á., Constructions and extensions of free and controlled additive relations, In: Th. M. Rassias (Ed.), Handbook of Functional Equations: Functional Inequalities, Springer Optim. Appl., 95(2014), 161-208.

[37] Gselmann E., Páles, Zs., Additive solvability and linear independence of the solutions of a system of functional equations, Acta Sci. Math. (Szeged), 82(2016), 101-110.

[38] Hyers, D.H., On the stability of the linear functional equation, Proc. Natl. Acad. Sci. USA, 27(1941), 222-224.

[39] Hyers, D.H., Rassias, Th.M., Approximate homomorphisms, Aequationes Math., 44(1992), 125-153.

[40] Hyers, D.H., Isac, G., Rassias, Th.M., Stability of Functional Equations in Several Variables, Birkhäuser, Boston, 1998.

[41] Hyers, D.H., Isac, G., Rassias, Th.M., On the asymptotic aspect of Hyers-Ulam stability of mappings, Proc. Amer. Math. Soc., 126(1998), 425-430.

[42] Jessen, B., Karpf, J., Thorup, A., Some functional equations in groups and rings, Math. Scand., 22(1968), 257-265.

[43] Jun, K.-W., Shin, D.-S., Kim, B.-D., On the Hyers-Ulam-Rassias stability of the Pexider equation, J. Math. Anal. Appl., 239(1999), 20-29.

[44] Jung, S.M., Hyers-Ulam-Rassias Stability of Functional Equations in Mathematical Analysis, Hadronic Press, Palm Harbor, Fl, 2001.

[45] Jung, S.M., Local stability of the additive functional equation, Glas. Mat. Ser. III, 38(2003), 45-55.

[46] Jung, S.M., Hyers-Ulam-Rassias Stability of Functional Equations in Nonlinear Analysis, Springer Optim. Appl. 48, New York, 2011.

[47] Jung, S.M., Moslehian, M.S., Sahoo, P.K., Stability of a generalized Jensen equation on restricted domains, J. Math. Inaqual., 4(2010), 191-206.

[48] Jung, S.M., Popa, D., Rassias, M.Th., On the stability of the linear functional equation in a single variable on complete metric groups, J. Global. Optim., 59(2014), 165-171.

[49] Kannappan, Pl., Functional Equations and Inequalities with Applications, Springer, Dordrecht, 2009.

[50] Kuczma, M., An Introduction to the Theory of Functional Equations and Inequalities, Polish Sci. Publ. and Univ. Ślaski, Warszawa, 1985.

[51] Kurepa, S., On some functional equations, Glas. Mat. Ser. III, 11(1956), 95-100.

[52] Losonczi, L., On the stability of Hossú's functional equation, Results Math., 29(1996), 305-310.

[53] Maksa, Gy., Páles, Zs., Hyperstability of a class of linear functional equations, Acta Math. Acad. Paedagog. Nyházi. (N.S.), 17(2001), 107-112. 
[54] Manar, Y., Elqorachi, E., Rassias, Th.M., On the generalized Hyers-Ulam stability of the Pexider equation on restricted domains, In: Rassias, Th. M. (Ed.), Handbook of Functional Equations, Stability Theory, Springer Optim. Appl., 96(2014), 279-299.

[55] Molaei, D., Najati, A., Hyperstability of the general linear equation on restricted domains, Acta Math. Hungar., 149(2016), 238-253.

[56] Moszner, Z., On the stability of functional equations, Aequationes Math., 77(2009), 3388.

[57] Moszner, Z., Stability has many names, Aequationes Math., 90(2016), 983-999.

[58] Najati, A., Molaee, D., Park, Ch., Hyperstability of a generalized Cauchy functional equation, J. Comput. Anal. Appl., 22(2017), 1049-1054.

[59] Najati, A., Rassias, Th.M., Stability of the Pexiderized Cauchy and Jensen's equations on restricted domains, Commun. Math. Anal., 8(2010), 125-135.

[60] Páles, Zs., Bounded solutions and stability of functional equations for two variable functions, Results Math., 26(1994), 360-365.

[61] Piszczek, M., Remark on hyperstability of the general linear equation, Aequationes Math., 88(2014), 163-168.

[62] Piszczek, M., Hyperstability of the general linear functional equation, Bull. Korean Math. Soc., 52(2015), 1827-1838.

[63] Pólya, Gy., Szegő, G., Aufgaben und Lehrsätze aus der Analysis I, Verlag von Julius Springer, Berlin, 1925.

[64] Rassias, J.M., On approximation of approximately linear mappings by linear mappings J. Funct. Anal., 46(1982), 126-130.

[65] Rassias, Th. M. On the stability of the linear mapping in Banach spaces, Proc. Amer. Math. Soc., 72(1978), 279-300.

[66] Rassias, Th.M., Stability and set-valued functions, In: Analysis and Topology, World Sci. Publ., River Edge, NJ, 1998, 585-614.

[67] Rassias, Th.M., The problem of S. M. Ulam for approximately multiplicative mappings, J. Math. Anal. Appl., 246(2000), 352-378.

[68] Rassias, Th.M., On the stability of functional equations and a problem of Ulam, Acta Appl. Math., 62(2000), 23-130.

[69] Rassias, Th.M., On the stability of functional equations in Banach spaces J. Math. Anal. Appl., 251(2000), 264-284.

[70] Rassias, Th.M., On the stability of functional equations originated by a problem of Ulam, Mathematica (Cluj), 44(2002), 39-75.

[71] Sánchez, F.C., Castillo, J.M.F., Banach space techniques underpinning a theory for nearly additive mappings, Dissertationes Math. (Rozprawy Mat.), 404(2002), 73 pp.

[72] Skof, F., Sull'approssimazione delle applicazioni localmente $\delta$-additive, Atti Accad. Sci. Torino, 117(1983), 377-389.

[73] Skof, F., Properietá locali e approssimazione di operatori, Rend. Semin. Mat. Fis. Milano, 53(1983), 113-129.

[74] Špakula, J., Zlatoš, P., Almost homomorphisms of compact groups, Illinois J. Math., 48(2004), 1183-1189.

[75] Stetkaer, H., Functional Equations on Groups, World Scientific, New Jersey, 2013.

[76] Száz, Á., Preseminormed spaces, Publ. Math. Debrecen, 30(1983), 217-224. 
[77] Száz, Á., The Hyers-Ulam and Hahn-Banach theorems and some elementary operations on relations motivated their set-valued generalizations, In: P. M. Pardalos, P. G. Georgiev and H. M. Srivastava (Eds.), Stability, Approximations, and Inequalities, Springer Optim. Appl. 68(2012), 631-705.

[78] Száz, Á., Two natural generalizations of cocycles, J. Int. Math. Virtual Inst., 6(2016), 66-86.

[79] Száz, Á., Remarks and problems at the conference on inequalities and applications, Hajdúszoboszló, Hungary, 2016, Tech. Rep., Inst. Math., Univ. Debrecen, 9(2016), 34 pp.

[80] Száz, Á., A natural Galois connection between generalized norms and metrics, Acta Univ. Sapientiae Math., 9(2017), 353-366.

[81] Székelyhidi, L., Stability properties of functional equations in several variables, Stochastica, 47(1995), 95-100.

[82] Székelyhidi, L., Ulam's problem, Hyers's solution - and to where they led, In: Th. M. Rassias (Ed.), Functional Equations and Inequalities, Math. Appl., 518, Kluwer Acad. Publ., Dordrecht, 2000, 259-285.

[83] Tabor, J., Tabor, J., Stability of the Cauchy functional equation in metric groupoids Aequationes Math., 76(2008), 92-104.

[84] Volkmann, P., Zur Stabilität der Cauchyschen und der Hosszúschen Funktionalgleichung, Seminar 40(1998), No. 1, 5 pp.

Árpád Száz

University of Debrecen

Institute of Mathematics

H-4002 Debrecen, Pf. 400, Hungary

e-mail: szaz@science.unideb.hu 